\title{
A method for the computation of turbulent polymeric liquids including hydrodynamic interactions and chain entanglements
}

\author{
Demosthenes Kivotides a \\ Department of Chemical Engineering, University of Strathclyde Glasgow
}

(Dated: November 18, 2016)

\begin{abstract}
An asymptotically exact method for the direct computation of turbulent polymeric liquids that includes (a) fully resolved, creeping microflow fields due to hydrodynamic interactions between chains, (b) exact account of (subfilter) residual stresses, (c) polymer Brownian motion, and (d) direct calculation of chain entanglements, is formulated. Although developed in the context of polymeric fluids, the method is equally applicable to turbulent colloidal dispersions and aerosols.
\end{abstract}

a On visit to Department of Aerospace, California Institute of Technology (GALCIT) 


\section{PROLOGUE}

Traditionally, the study of polymeric liquids $[1,2]$ (and similarly of colloidal dispersions $[3,4])$ involves two major strains of thought. On the one hand, there is the viscoelastic fluid dynamics approach [5-7], that models complex fluids as continuum field theories, by employing a suitable constitutive law. Due to its relative simplicity and affinity with standard fluid dynamical investigations, this approach is particularly suitable for the analysis of complicated flow phenomena including instabilities and turbulence [8-10]. However, such studies are usually limited to dilute polymer systems, since dense polymer flows necessarily involve entanglements between polymer chains, and the effects of the latter on elastic stresses levels are difficult to accurately capture with standard constitutive laws $[11,12]$. Another, equally important, limitation of the classical field theoretic approach is that the employed constitutive laws originate in rheological flows that are either simple elongational/shear flows, or involve periodic unsteady effects (see particularly lucid discussions of these in $[11,13])$. The applicability of rheological constitutive laws to fully developed turbulent fluctuation fields is not straightfoward, since the latter are non-Gaussian and highly intermittent [14-16], hence the polymer chains find themselves interacting with velocity fields of a much higher degree of unstructured unsteadiness than usually is the case in rheology $[17,18]$.

The need for a constitutive law is bypassed via mesoscopic modeling of polymeric liquids [19]. In this formulation, the solvent is described via the Navier-Stokes equation, and is coupled with the polymer chains that are modeled by some version of the bead-spring model $[12,20,21]$. Due to the mesoscopic character of the modeling, the polymer chains interact via effective intermolecular potentials, and undergo Brownian motion. Such hybrid fluid-chains formulation has many advantages over the aforementioned fully continuum approach: (a) there is no need for a constitutive law, since elastic effects in the flow are taken into account from first principles via chain elasticity, and the explicit coupling of the chains with the flow field. It is important to note that, in order for this statement to be valid in non-dilute systems, one needs to employ a version of the bead-spring model that allows the formation of entanglements between chains and the calculation of their implications on elastic stress levels, (b) since information about the locations of polymer chains and fluid vortical structures is made available by the model, the detailed physics of turbulence-polymer inter- 
actions in grid turbulence [22] and turbulent boundary layers (excellently discussed in the context of polymer drag reduction in [23]) can be studied. Notably, even in dilute systems, such turbulence-polymer couplings would most probably be entwined with both chain entanglement and hydrodynamic-interactions effects between chains in locally polymer-dense areas, as is the case, for example, in between turbulent coherent vortices where polymers might be expected to concentrate [14, 24]. Similar ideas are also valid for turbulent flows in colloidal dispersions and aerosols [25], that feature particle aggregation/clustering phenomena $[26,27]$ which require the capturing of hydrodynamic interactions between particles at high concentration areas, as is the case, for example, during rain initiation processes [28-30].

Therefore, there is a need for mesoscopic, physical formulations/numerical methods that allow the direct computation of turbulent polymeric (and colloidal) fluids. Such methods have to overcome a number of challenges: (a) the forcing of the fluid by the particles is delta-function type (i.e., pointwise), hence standard methods for computational fluid dynamics need an extremely fine grid in order to capture the microscopic flow field in between the particles that corresponds to their hydrodynamic interactions, (b) efforts to average the forced Navier-Stokes equations in order to overcome this problem, lead, due to nonlinearity, to the appearance of subgrid scale stresses that need to be taken into account via some type of modeling, (c) the accompanying numerical method needs to handle the Brownian, i.e., stochastic motion of polymer and colloidal particles; this adds an additional level of complexity to standard computational methods for suspensions [31], (d) in polymeric liquids, the formulation and numerics need to describe the formation and dynamics of entanglements between macromolecular chains, in order for the approach to be applicable to arbitrary polymer volume fractions. In this paper, I formulate a computational method that addresses all these issues, and, moreover, in the limit of very small particle sizes, it becomes asymptotically exact.

\section{MESOSCOPIC DESCRIPTION OF POLYMERIC FLOWS}

As discussed above, within the mesoscopic framework, a solvent/continuum is coupled to a polymer/particle system. Hence, the solvent is fully described by an (incompressible) 
velocity flow field $\mathbf{u}(\mathbf{x}, t)$ at position $\mathbf{x}$ and time $t$, and a fluid pressure $p(\mathbf{x}, t)$. In order to avoid a complicated mathematical formalism, I am going to describe the polymers directly at the bead-spring model level $[20,21]$ (i.e., I am going to avoid a description in terms of one-dimensional elastic curves). An advantage of such a choice is that the connection of the formulation with both its polymer computational implementations and colloidal-dispersions applications is significantly more transparent. Hence, in the context of a bead spring model, each of the $N_{c}$ chains in the computational domain is discretized into $N_{b}$ spherical beads (thus the total number of particles in the system is equal to $N=N_{c} N_{b}$ ). Consequently, a key polymer variable is the $N$-dimensional bead-position vector $\mathbf{R}_{b}=\left(\mathbf{r}^{1}, \mathbf{r}^{2}, \ldots, \mathbf{r}^{N}\right)$. In the following, I employ tensor notation such as $u_{i}, r_{j}^{k}, r_{i}^{k^{\prime}}$ where $i, j=1, \ldots, 3$ are indices denoting the three spatial directions, and $k, k^{\prime}=1, \ldots, N$ are indices denoting polymer beads. Repeated indices are always summed, unless stated otherwise.

The motion of the solvent (fluid) obeys the forced Navier-Stokes equations for the fluid velocity $\mathbf{u}(\mathbf{x}, t)$ :

$$
\begin{gathered}
\frac{\partial u_{i}}{\partial x_{i}}=0 \\
\rho \frac{\partial u_{i}}{\partial t}+\rho \frac{\partial\left(u_{i} u_{j}\right)}{\partial x_{j}}+\frac{\partial p}{\partial x_{i}}-\mu \frac{\partial^{2} u_{i}}{\partial x_{j} \partial x_{j}}-{ }^{d} F_{i}^{k} \delta\left(\mathbf{x}-\mathbf{r}^{k}\right)=0
\end{gathered}
$$

where $\rho$ is the fluid's mass density, $\mu$ is the fluid's dynamic viscosity, and ${ }^{d} F_{i}^{k}$ is the drag force exerted by the polymer bead $k$ onto the fluid. There is an implied summation over the bead index $k$. Since this is a coupling force, it appears with exactly the opposite sign in the chain dynamics equation. As discussed above, the correct account of this force requires the resolution of the hydrodynamic interactions between the various polymer sections.

The polymers obey Brownian (stochastic) dynamics. In order to incorporate entanglements in the polymer physics, I am going to follow here the entanglement model of [20, 21]. The model is best explained by returning momentarily to the continuum description of polymer dynamics, i.e., by considering the ensemble of $N_{c}$ elastic strings that is collectively called $\mathcal{L}$. Then the motion of a point $\mathbf{r}_{\mathcal{L}} \in \mathcal{L}$ is described by the equation $[20,21]$

$$
{ }^{\mathbf{i}} \mathbf{f}+{ }^{\mathbf{e}} \mathbf{f}+{ }^{\mathbf{m}_{\mathbf{f}}}+{ }^{\mathbf{c}} \mathbf{f}+{ }^{\mathbf{d}} \mathbf{f}+{ }^{\mathrm{t}} \mathbf{f}=0
$$




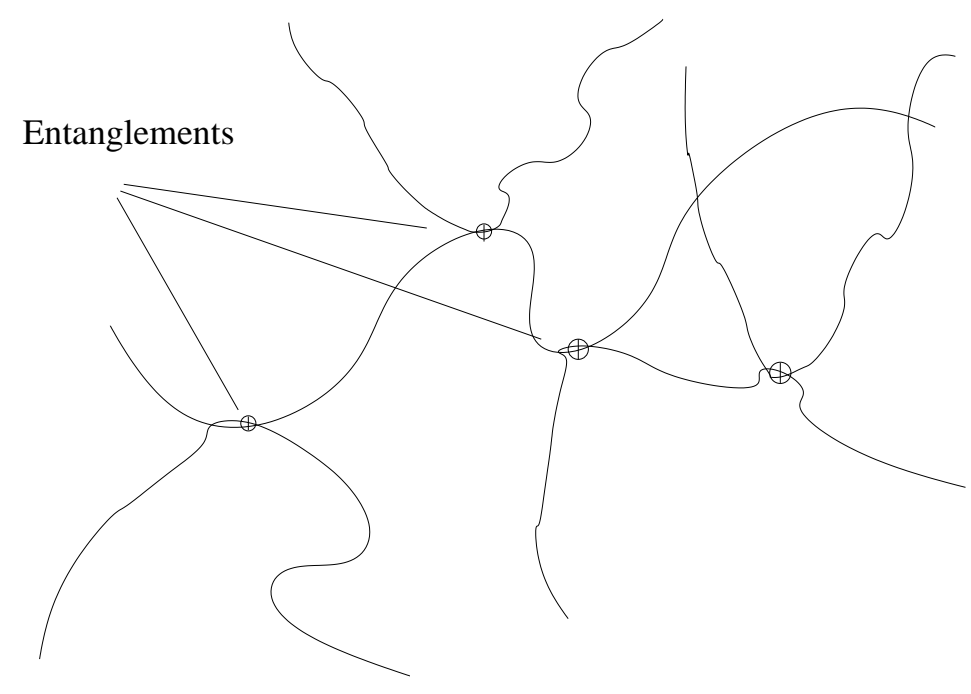

FIG. 1. Entanglements between chains are of key dynamical importance; by obstructing polymer motion they are responsible for elastic stress augmentation

where ${ }^{\text {if }}=\mu_{p} \mathrm{~d}^{2} \mathbf{r}_{\mathcal{L}} / \mathrm{d} t^{2}$ (with $\mu_{p}$ been the polymer mass per unit length) is the inertial force, ${ }^{\mathbf{e}} \mathbf{f}$ is the elastic force, ${ }^{\mathbf{m}_{\mathbf{f}}}$ is the intermolecular force, ${ }^{\mathbf{d}_{\mathbf{f}}}$ is the hydrodynamic drag force, and ${ }^{\mathbf{t}} \mathbf{f}$ is the Brownian thermal force (all per unit length). The contact force ${ }^{\mathbf{c}} \mathbf{f}$ was introduced in [20] in order to ensure that the polymer chains cannot pass through each other. This cannot be accomplished by the coarse-grained excluded volume force ${ }^{\mathbf{m}_{\mathbf{f}}} \mathbf{\text { since, }}$, due to mesoscopic coarse-graining, the latter corresponds to a soft (often exponential) potential that reproduces the effects of the microscopic intermolecular forces on average, hence it does not necessarily instantaneously preserve the topological integrity of $\mathcal{L}$. As shown in [20], this leads to detrimental physical effects in the rheology of knotted (bio)polymers. The force ${ }^{{ }^{\mathbf{c}} \mathbf{f}}$ (that eliminates these spurious effects) can be considered to be the gradient of a potential $\phi^{c}\left(\mathbf{r}, \mathbf{r}^{\prime}\right)=\phi^{c}\left(\mathbf{r}-\mathbf{r}^{\prime}\right)=g \delta\left(\mathbf{r}-\mathbf{r}^{\prime}\right)$, where $\mathbf{r}, \mathbf{r}^{\prime} \in \mathcal{L}$. The positive constant $g$ is taken to be adequately large in order to capture and preserve chain entanglements $\mathbf{r}_{e} \in \mathcal{L}$ (i.e., contact points between chains) that are defined as

$$
\mathbf{r}_{e}=\int_{\mathcal{L}-V_{\epsilon}\left(\mathbf{r}_{\mathcal{L}}\right)} d\left|\mathbf{r}_{\mathcal{L}}\right| \mathbf{r}_{\mathcal{L}} \delta\left(\left|\mathbf{r}_{e}-\mathbf{r}_{\mathcal{L}}\right|\right)
$$

where $V_{\epsilon}\left(\mathbf{r}_{\mathcal{L}}\right) \in \mathcal{L}$ is a small neighborhood around $\mathbf{r}_{\mathcal{L}}$. A schematic demonstration of this definition is depicted in Fig.1. So in analogy with $\mathbf{R}_{b}$, a position vector for all $N_{e}$ entanglements in the system can be defined $\mathbf{R}_{e}=\left(\mathbf{r}_{e}^{1}, \mathbf{r}_{e}^{2}, \ldots, \mathbf{r}_{e}^{N_{e}}\right)$. The numerical/computational implementation of force ${ }^{\mathbf{c}} \mathbf{f}$ is highly technical. The particulars of the method which is an 
evolved version of the geometric approach of [32] are discussed in [20,21]. For the purposes of present exposition, it suffices to write the final equation governing bead-motion

$$
{ }^{i} F_{i}^{k}+{ }^{e} F_{i}^{k}+{ }^{m} F_{i}^{k}+{ }^{d} F_{i}^{k}+{ }^{t} F_{i}^{k}=0,
$$

where ${ }^{i} F_{i}^{k}=m_{b} \mathrm{~d}^{2} r_{i}^{k} / \mathrm{d} t^{2}$ (with $m_{b}$ been the mass of each polymer bead) is the inertial force, and the physical meaning of the other forces is as above, only now they refer to total forces acting on polymer sections corresponding to individual beads. Notably, the drag force ${ }^{d} F_{i}{ }^{k}$ appears with opposite sign than in the Navier-Stokes equation. A key observation is that the contact force is not present, but its effects are taken into account in the expression for the elastic force ${ }^{\mathbf{e}} \mathbf{F}^{k}={ }^{\mathbf{e}} \mathbf{F}^{k}\left(\mathbf{R}_{b}, \mathbf{R}_{e}\right)$, which now also depends on the number and position of chain entanglements [20, 21]. Consequently, any method incorporating the above beadspring model automatically includes chain-entanglement effects.

\section{METHOD FOR THE COMPUTATION OF TURBULENT POLYMERIC LIQUIDS}

There are some strict requirements for such a method. First of all, it needs to compute the drag force ${ }^{\mathrm{d}} \mathbf{F}^{k}$ whilst resolving the hydrodynamic interactions between beads. Since the latter are caused by pointwise forcings, the grid requirements for their direct computation via standard finite volume solvers are (complexity wise) prohibitive. Moreover, any approach capable of resolving the microscopic flow in between the beads needs to simultaneously allow the incorporation of Brownian effects into the chain dynamics. At the same time, the method ought to resolve all turbulent fluctuations from first principles. Finally, the whole formulation must be asymptotically exact in the limit of very small particle size. Next, I provide an overview of the method, by presenting the dynamical equations and the corresponding algorithmic steps, before I discuss in greater detail the specific elements of the new formulation.

\section{Summary of dynamical equations}

In a nutshell, the method solves the coupled dynamics of a filtered Navier-Stokes equation for the fluid, and a Coarse-Grained Molecular Dynamics, Langevin equation for the polymer 
chains. In order to compute the chain-induced hydrodynamic-interactions flow field that is required for the evaluation of both the drag force in the Langevin equation and the subfilter flow field in the filtered Navier-Stokes equation, the method incorporates the solution of a Stokes equation that models the (hydrodynamic interactions) creeping flow field that (at each instant) is in equilibrium with the large (above the filter) scale flow field. The key equations are

$$
\begin{aligned}
& \frac{\partial \bar{u}_{i}}{\partial x_{i}}=0 \\
& \rho \frac{\partial \bar{u}_{i}}{\partial t}+\rho \frac{\partial\left(\bar{u}_{i} \bar{u}_{j}\right)}{\partial x_{j}}+\frac{\partial \bar{p}}{\partial x_{i}}+\rho \frac{\partial\left(\overline{u_{i}^{\prime} u_{j}^{\prime}}\right)}{\partial x_{j}}-\mu \frac{\partial^{2} \bar{u}_{i}}{\partial x_{j} \partial x_{j}}-{ }^{d} F_{i}^{k} G_{\xi}\left(\mathbf{x}-\mathbf{r}^{k}\right)=0 \\
& { }^{i} F_{i}{ }^{k}+{ }^{e} F_{i}^{k}+{ }^{m} F_{i}{ }^{k}+{ }^{d} F_{i}{ }^{k}+{ }^{t} F_{i}^{k}=0, \\
& \frac{\partial u_{i}^{\mathcal{S}}}{\partial x_{i}}=0 \\
& \frac{\partial p^{\mathcal{S}}}{\partial x_{i}}-\mu \frac{\partial^{2} u_{i}^{\mathcal{S}}}{\partial x_{j} \partial x_{j}}-{ }^{d} F_{i}^{k} \delta\left(\mathbf{x}-\mathbf{r}^{k}\right)=0 \\
& \overline{u_{i}^{\prime} u_{j}^{\prime}}(\mathbf{x})=\overline{\left(u_{l}^{\mathcal{S}}\right)_{i}\left(u_{l}^{\mathcal{S}}\right)_{j}}(\mathbf{x})=\int_{V}\left(u_{l}^{\mathcal{S}}\right)_{i}\left(\mathbf{x}-\mathbf{x}^{\prime}\right)\left(u_{l}^{\mathcal{S}}\right)_{j}^{\prime}\left(\mathbf{x}-\mathbf{x}^{\prime}\right) G_{\xi}\left(\mathbf{x}^{\prime}\right) \mathrm{d} \mathbf{x}^{\prime},
\end{aligned}
$$

where (5) and (6) are the filtered Navier-Stokes equations, (7) is the Langevin equation for the polymer beads, (8) and (9) are the creeping flow, Stokes equations, and (10) describes the residual stress in (6) in terms of the local part $u_{l}^{\mathcal{S}}$ of the Stokes velocity field $u^{\mathcal{S}}=$ $u_{l}^{\mathcal{S}}+u_{g}^{\mathcal{S}}$ (where $u_{g}^{\mathcal{S}}$ is the global part). The various terms in these equations are gathered for convenience in Table I. Their detailed meaning is discussed in the sections that follow.

\section{Filtering the forced Navier-Stokes equation}

In order to start unraveling the approach, I focus first on fluid momentum, and note that irrespective of the specific physics of turbulent fluctuations, one can always define for the latter a length scale $\xi$ ( $k_{\xi}$ in spectral space) for which the corresponding Reynolds number is equal to unity, or, in other words, below $\xi$ there cannot be any inertial flow fluctuations (although there can be fluctuations due to a complicated creeping flow velocity field). Since the aim here is to fully resolve the turbulence scales, one can filter the forced Navier-Stokes equation at the $\xi$ scale, without any loss of accuracy, as far as, turbulence physics are 


\begin{tabular}{|c|c|}
\hline $\bar{u}_{i}$ & $i$ component of filtered fluid velocity \\
\hline $\bar{p}$ & filtered fluid pressure \\
\hline$u_{i}^{\prime}$ & $i$ component of residual (subfilter) fluid velocity \\
\hline$\overline{u_{i}^{\prime} u_{j}^{\prime}}$ & residual (subfilter) fluid stress \\
\hline$G_{\xi}$ & filter function \\
\hline$\left(\mathbf{R}_{b}(t), \mathbf{R}_{e}(t)\right)$ & bead/particle and entanglement positions \\
\hline${ }^{d} F_{i}{ }^{k}$ & $i$ component of dissipative drag force on bead/particle $k$ \\
\hline${ }^{i} F_{i}{ }^{k}$ & $i$ component of inertial force on bead/particle $k$ \\
\hline${ }^{e} F_{i}{ }^{k}$ & $i$ component of elastic force on bead $k$ \\
\hline${ }^{m} F_{i}{ }^{k}$ & $i$ component of intermolecular force on bead/particle $k$ \\
\hline${ }^{t} F_{i}{ }^{k}$ & $i$ component of (thermal) fluctuation force on bead/particle $k$ \\
\hline$u_{i}^{\mathcal{S}}$ & $i$ component of Stokes flow field \\
\hline$p^{\mathcal{S}}$ & pressure enforcing Stokes flow incompressibility \\
\hline$\left(u_{l}^{\mathcal{S}}\right)_{i}$ & $i$ component of local part of Stokes flow field \\
\hline$\left(u_{g}^{\mathcal{S}}\right)_{i}$ & $i$ component of global part of Stokes flow field \\
\hline
\end{tabular}

TABLE I.

Nomenclature of various quantities appearing in the dynamical equations

concerned (assuming, of course, that $\xi=\Delta x$, where $\Delta x$ is the computational grid size of the Navier-Stokes solver). Any function $G_{\xi}\left(\mathbf{x}^{\prime}\right)$ that is even in $\mathbf{x}^{\prime}$, integrates to unity over all space, is of order $1 / \xi^{N}$ at the origin (where $N$ is the space dimension), and is $G_{\xi}\left(\mathbf{x}^{\prime}\right) \ll 1$ for $\left|\mathbf{x}^{\prime}\right| \gg \xi$ is a suitable filter. Well known filters are the box, Gaussian and sharp spectral filters [24]. For example, noting that three-dimensional filter functions are related to their onedimensional versions via the formula $G_{\xi}\left(\mathbf{x}^{\prime}\right)=\prod_{i=1,3} G_{\xi}\left(x_{i}^{\prime}\right)$, the three-dimensional Gaussian filter reads

$$
G_{\xi}\left(\mathbf{x}^{\prime}\right)=\frac{1}{\pi^{3 / 2} \xi^{3}} e^{-\frac{\left|\mathbf{x}^{\prime}\right|^{2}}{\xi^{2}}}
$$

and the three-dimensional sharp spectral filter reads

$$
G_{\xi}\left(\mathbf{x}^{\prime}\right)=\frac{\sin \left(\pi x_{1}^{\prime} / \xi\right) \sin \left(\pi x_{2}^{\prime} / \xi\right) \sin \left(\pi x_{3}^{\prime} / \xi\right)}{\pi^{3} x_{1}^{\prime} x_{2}^{\prime} x_{3}^{\prime}} .
$$


The filtered fluid velocity becomes

$$
\overline{\mathbf{u}}(\mathbf{x}) \equiv \mathbf{u}_{\xi}(\mathbf{x})=\int_{V} \mathbf{u}\left(\mathbf{x}-\mathbf{x}^{\prime}\right) G_{\xi}\left(\mathbf{x}^{\prime}\right) \mathrm{d} \mathbf{x}^{\prime}
$$

and one can write $\mathbf{u}=\overline{\mathbf{u}}+\mathbf{u}^{\prime}$. It is important to note that although the filtering and differentiation operators commute, it is in general $\overline{\overline{\mathbf{u}}} \neq \overline{\mathbf{u}}$. However, there are filters, and the sharp spectral filter is an example, where $\overline{\overline{\mathbf{u}}}=\overline{\mathbf{u}}$, hence $\overline{\mathbf{u}^{\prime}}=0$. Assuming that such $a$ filter function is employed, we obtain the filtered Navier-Stokes equations for the solenoidal velocity field $\overline{\mathbf{u}}(\mathbf{x})$

$$
\begin{gathered}
\frac{\partial \bar{u}_{i}}{\partial x_{i}}=0 \\
\rho \frac{\partial \bar{u}_{i}}{\partial t}+\rho \frac{\partial\left(\bar{u}_{i} \bar{u}_{j}\right)}{\partial x_{j}}+\frac{\partial \bar{p}}{\partial x_{i}}+\rho \frac{\partial\left(\overline{u_{i}^{\prime} u_{j}^{\prime}}\right)}{\partial x_{j}}-\mu \frac{\partial^{2} \bar{u}_{i}}{\partial x_{j} \partial x_{j}}-{ }^{d} F_{i}^{k} G_{\xi}\left(\mathbf{x}-\mathbf{r}^{k}\right)=0,
\end{gathered}
$$

where, due to the pointwise nature of the forcing term, the delta function has now been replaced by the filter function, and the gradient of the so called residual stress $\tau_{i j}^{R} \equiv-\rho \overline{u_{i}^{\prime} u_{j}^{\prime}}$ has appeared. This stress is a direct consequence of the convective nonlinearity in the inertial force, and it ought not to be confused with the Reynolds stresses that are the result of probabilistic (ensemble) averaging rather than spatial filtering. Indeed, the solution of this equation is a fully developed turbulent flow that includes all the fluctuation scales. One can apply probabilistic averaging to it in order to recover the Reynolds stresses, or any higher order statistics as desired. The filtered equation can be solved numerically with standard finite volume, projection methods [36, 37], hence, at this point, the key difficulties are the computation of the forcing term by taking into account hydrodynamic interactions between chains, and the accurate (i.e., without resort to modeling) calculation of the residual stress $\tau_{i j}^{R}$. These issues are addressed next.

\section{Creeping microflow and polymer motion}

In order to simplify the exposition and facilitate its computational implementation, I embed it within a hypothetical computational algorithm: at time step $n$, i.e., at time $t=n \Delta t$, the filtered velocity field $\overline{\mathbf{u}}(\mathbf{x}, t)$, and the bead and entanglement positions $\left(\mathbf{R}_{b}(t), \mathbf{R}_{e}(t)\right)$ are available. Since the aim is to resolve the turbulence fluctuations, the temporal time step $\Delta t$ is chosen to be of the order of the fastest eddy turnover times in the turbulent fluid. Now, due to small polymer-bead (and colloid) diameters and polymer thickness, it is expected 
that the microflow around polymer chains is always going to be a creeping flow that can be described by Stokesian Dynamics [33]. Since, due to its diffusive nature, a creeping flow establishes itself essentially instantaneously with respect to the much slower inertial turbulence fluctuations [13], the hydrodynamic interactions and the accompanying viscous drag on polymer chains correspond to Stokes flow modes superposed on large scale turbulence. In other words, without loss of accuracy, the turbulence can be considered "frozen" during the time required for the creeping flow regime to reach a steady state (or else to equilibrate) with respect to its instantaneous velocity fluctuations $\overline{\mathbf{u}}(\mathbf{x}, t)$ that are resolved by the filtered Navier-Stokes equation. Similarly, the Stokes relaxation time $\tau=m_{b} /(6 \pi \mu a)$ (where $a$ is the polymer-bead radius) is very small, so, since the aim is to accurately resolve the dynamics of inertial turbulence fluctuations that evolve at much larger time scales, one can solve the polymer dynamics in the diffusive limit $[34,35]$, when the inertial force has settled to zero.

Implementing these in the balance of forces in the polymer dynamics, one obtains

$$
{ }^{d} F_{i}^{k}=\zeta_{i j}^{k k^{\prime}} v_{j}^{k^{\prime}}=-{ }^{e} F_{i}^{k}-{ }^{m} F_{i}^{k}-{ }^{t} F_{i}^{k}
$$

which indicates that, taking into account the very small Stokes time, it is only because of the presence of elastic and intermolecular forces that the chain velocites are allowed to "slip" relative to $\overline{\mathbf{u}}$, hence, to also experience a drag force. Here, $\zeta_{i j}^{k k^{\prime}}$ is the symmetric $3 N \times 3 N$ hydrodynamic-friction matrix $\boldsymbol{\zeta}$. According to this notation, this matrix is composed by $N \times$ $N, 3 \times 3$ matrices. Hence, the upper $k k^{\prime}$ indices point to the particular $3 \times 3$ hydrodynamicfriction submatrix that corresponds to beads $k$ and $k^{\prime}$, and the lower $i j$ indices help pick up one of the 9 elements of the $k k^{\prime}$ submatrix. Moreover, $v_{j}^{k^{\prime}}$ is the $j$ component of the Stokesian velocity $\mathbf{v}^{k^{\prime}}$ induced at bead-position $\mathbf{r}^{k^{\prime}}$ by the totality of coupling forces between the polymer chains and the fluid. Notably, the non-diagonal nature of the hydrodynamicfriction matrix automatically takes into account the hydrodynamic interactions between beads. I remark for clarity that, in the $\zeta_{i j}^{k k^{\prime}} v_{j}^{k^{\prime}}$ expression, two summations over the $j$ and $k^{\prime}$ repeated indices are implied. Moreover, as discussed above, the total flow velocity at bead location $\mathbf{r}^{k}$ is equal to $\overline{\mathbf{u}}\left(\mathbf{r}^{k}\right)+\mathbf{v}^{k}$. For completeness, I also mention here the corresponding equation for fluid momentum

$$
\rho \frac{\partial \bar{u}_{i}}{\partial t}+\rho \frac{\partial\left(\bar{u}_{i} \bar{u}_{j}\right)}{\partial x_{j}}+\frac{\partial \bar{p}}{\partial x_{i}}+\rho \frac{\partial\left(\overline{u_{i}^{\prime} u_{j}^{\prime}}\right)}{\partial x_{j}}-\mu \frac{\partial^{2} \bar{u}_{i}}{\partial x_{j} \partial x_{j}}-\zeta_{i j}^{k k^{\prime}} v_{j}^{k^{\prime}} G_{\xi}\left(\mathbf{x}-\mathbf{r}^{k}\right)=0
$$


where now three summations over $j, k$ and $k^{\prime}$ repeated indices are implied in the expression for the drag force. Employing the balance of forces in the polymer dynamics, the drag force in the filtered Navier-Stokes equation can be replaced by the sum of elastic and intermolecular forces. It is important to note that, despite this substitution, the turbulence dynamics are directly informed by hydrodynamic interaction effects, since the elastic and intermolecular forces depend only on the bead and entanglement positions $\left(\mathbf{R}_{b}(t), \mathbf{R}_{e}(t)\right)$, and full hydrodynamic-interaction effects are taken into account when new values for $\left(\mathbf{R}_{b}(t), \mathbf{R}_{e}(t)\right)$ are computed. This feature of the method is discussed next.

Returning to the hypothetical computational algorithm, and keeping in mind the above formalism, the first task within a numerical time step is to update $\left(\mathbf{R}_{b}(t), \mathbf{R}_{e}(t)\right)$. This is going to be achieved by taking into account (in an accurate way) the hydrodynamic interactions between particles, as well as Brownian motion effects. Moreover, the polymer numerical method (just like the projection methods employed for Navier-Stokes dynamics) needs to be suitable for bounded computational domains with arbitrary boundary conditions. As discussed above, it suffices to compute the Stokes flow $\mathbf{u}^{\mathcal{S}}(\mathbf{x})$ that is in equilibrium with the numerical large scale turbulent velocity $\overline{\mathbf{u}}(\mathbf{x})$

$$
\begin{gathered}
\frac{\partial u_{i}^{\mathcal{S}}}{\partial x_{i}}=0 \\
\frac{\partial p^{\mathcal{S}}}{\partial x_{i}}-\mu \frac{\partial^{2} u_{i}^{\mathcal{S}}}{\partial x_{j} \partial x_{j}}-{ }^{d} F_{i}{ }^{k} \delta\left(\mathbf{x}-\mathbf{r}^{k}\right)=0
\end{gathered}
$$

where $p^{\mathcal{S}}(\mathbf{x})$ is the pressure field required in order to make $\mathbf{u}^{\mathcal{S}}(\mathbf{x})$ incompressible. There are a number of methods available [34, 35, 38], however, I am going to base the discussion on the approach of [35], since this method was employed in the entangled polymer dynamics computation of [21], hence the latter reference could be consulted for various numerical and computational details, as required. I am presenting here only the method aspects that are relevant in the context of the present approach. After defining the hydrodynamic force density $\boldsymbol{\theta}(\mathbf{x})={ }^{d} \mathbf{F}^{k} \delta\left(\mathbf{x}-\mathbf{r}^{k}\right), \boldsymbol{\theta}(\mathbf{x})$ is written as the sum of two contributions $\boldsymbol{\theta}(\mathbf{x})=$ $\boldsymbol{\theta}_{l}(\mathbf{x})+\boldsymbol{\theta}_{g}(\mathbf{x})$, where the "local" density is $\boldsymbol{\theta}_{l}(\mathbf{x})={ }^{d} \mathbf{F}^{k}\left[\delta\left(\mathbf{x}-\mathbf{r}^{k}\right)-h\left(\mathbf{x}-\mathbf{r}^{k}\right)\right]$, and the "global" density is $\boldsymbol{\theta}_{g}(\mathbf{x})={ }^{d} \mathbf{F}^{k}\left[h\left(\mathbf{x}-\mathbf{r}^{k}\right)\right]$. The screening function $h$ satisfies the condition $\int_{V} h(\mathbf{x}) d \mathbf{x}=1$, where the integral is over all space and not just over the computational domain. The force density $\boldsymbol{\theta}_{l}(\mathbf{x})$ produces a local contribution to the velocity field $\mathbf{u}_{l}^{\mathcal{S}}(\mathbf{x})$, 


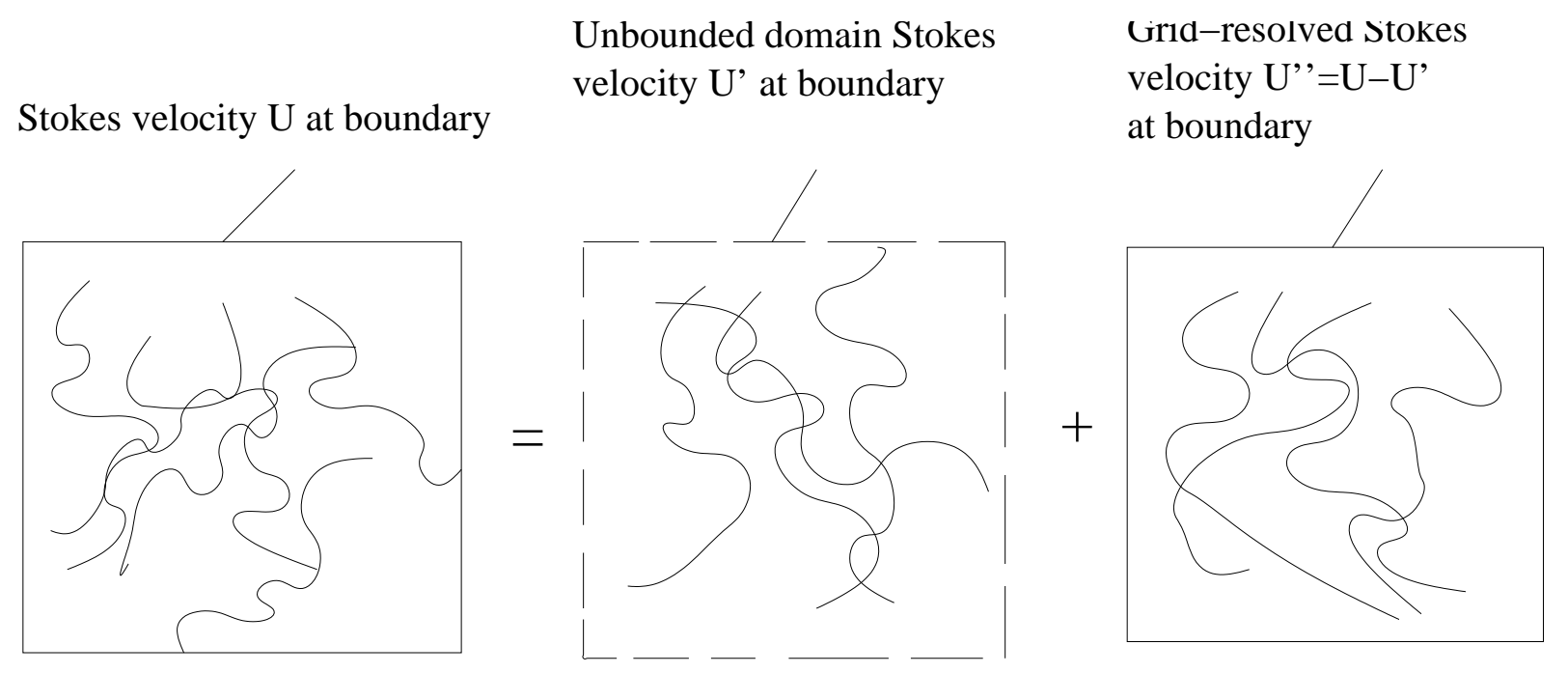

FIG. 2. Taking advantage of the linearity of the Stokes equation, one can satisfy its boundary conditions by summing an (unbounded-domain) Stokeslet, and a grid-resolved, bounded-domain Stokes flow satisfying appropriate boundary conditions

because the screening function depends on a parameter $\alpha$ that defines a length scale $\alpha^{-1}$ beyond which $\mathbf{u}_{l}^{\mathcal{S}}(\mathbf{x})$ decays exponentially to zero. In particular, $\mathbf{u}_{l}^{\mathcal{S}}(\mathbf{x})=\mathbf{H}_{l}\left(\mathbf{x}-\mathbf{r}^{k}\right) \cdot{ }^{d} \mathbf{F}^{k}$, where $\mathbf{H}_{l}(\mathbf{x})$ is composed of the free space stokeslet (corresponding to the delta function part) minus a smoothed free space stokeslet obtained from the analytical solution of the Stokes equations with the forcing term given by the function $\boldsymbol{\theta}_{g}(\mathbf{x})$ [35]. The force density $\boldsymbol{\theta}_{g}(\mathbf{x})$ corresponds to a global velocity field $\mathbf{u}_{g}^{\mathcal{S}}(\mathbf{x})$ that varies on the scale $\alpha^{-1}$, hence it can be solved in bounded domains, with finite volume solvers and suitable boundary conditions [21]. Since by linearity $\mathbf{u}^{\mathcal{S}}(\mathbf{x})=\mathbf{u}_{l}^{\mathcal{S}}(\mathbf{x})+\mathbf{u}_{g}^{\mathcal{S}}(\mathbf{x})$, the boundary conditions of $\mathbf{u}_{g}^{\mathcal{S}}(\mathbf{x})$ can be chosen appropriately so that, when added to the $\mathbf{u}_{l}^{\mathcal{S}}(\mathbf{x})$ values on the boundary, the physical boundary conditions for $\mathbf{u}^{\mathcal{S}}$ are satisfied. This is schematically shown in Fig.2. In the context of the present method, it is important to note that, (a) $\mathbf{u}_{l}^{\mathcal{S}}(\mathbf{x})$ includes in an analytical way the short-range hydrodynamic interaction effects that it is practically impossible to compute with finite-volume, turbulent flow solvers. Notably, $\mathbf{u}^{\mathcal{S}}\left(\mathbf{r}^{k}\right) \equiv \mathbf{v}^{k}$, i.e., the Stokes flow velocity at polymer-bead position $\mathbf{r}^{k}$. Hence, when the velocity $\overline{\mathbf{u}}\left(\mathbf{r}^{k}\right)+\mathbf{u}^{\mathcal{S}}\left(\mathbf{r}^{k}\right)$ is employed in order to advect the polymer chains, the direct incorporation of full hydrodynamic effects into chain motion is achieved, (b) the scale $\alpha^{-1}$ is not free to choose (as in rheology) but ought to be equal to the filtering scale $\xi$ above, i.e., $\xi \alpha=1$. Why this must be the case is going to be explained later on. 
There is a final issue with Brownian motion effects. In particular, the method just outlined makes available the Stokes velocity field at polymer-bead positions, $v_{i}^{k}=\mu_{i j}^{k k^{\prime} d} F_{j}^{k^{\prime}}$, where $\mu_{i j}^{k k^{\prime}}$ is the mobility matrix, i.e., the inverse of the hydrodynamic-friction matrix, $\boldsymbol{\mu}=\boldsymbol{\zeta}^{-1}$. Therefore, no explicit computation of $\boldsymbol{\mu}$ takes place, and this is problematic in the context of calculating Brownian motion effects, where knowledge of this matrix is required [33]. Hence, one needs to employ Fixman's method [39, 40] for solving stochastic differential equations, since the latter is a "matrix-free" method, requiring only matrix-vector products, not the $\boldsymbol{\mu}$ matrix itself. In this way, the part of the method that updates $\left(\mathbf{R}_{b}(t), \mathbf{R}_{e}(t)\right)$ is now complete.

\section{Computation of residual stresses in the fluid momentum equation}

Up till this point, a formulation of mesoscopic polymeric fluid dynamics has been developed, incorporating the governing equations of turbulence and polymer physics. The filtered velocity $\overline{\mathbf{u}}(\mathbf{x}, t)$, and the bead-entanglement positions $\left(\mathbf{R}_{b}(t), \mathbf{R}_{e}(t)\right)$ have been identified as the main variables. A method for computing the latter including hydrodynamic interactions, entanglements and Brownian motion effects has been described. The filtered Navier-Stokes equations for $\overline{\mathbf{u}}(\mathbf{x}, t)$ can be solved with finite volume, projection techniques. However, an inspection shows that a method for calculating the residual stresses $\tau_{i j}^{R} \equiv-\rho \overline{u_{i}^{\prime} u_{j}^{\prime}}$ is needed. This can be done in an exact way, without introducing any approximations. Indeed, since the filtering length scale is the inertial fluctuations cut-off $\xi$, turbulence is not supposed to contribute to $\tau_{i j}^{R}$. Moreover, as mentioned above, by choosing $\xi \alpha=1$ when determining the scale of variation $\alpha^{-1}$ of the global Stokesian flow field $\mathbf{u}_{g}^{\mathcal{S}}(\mathbf{x})$, the latter is enforced to vary on the same scales as turbulence, hence, it cannot either contribute to $\tau_{i j}^{R}$. Therefore, the only velocity field that can create subfilter scale fluctuations is the local Stokesian flow field $\mathbf{u}_{l}^{\mathcal{S}}(\mathbf{x})$. This is plausible, since, by construction, this is the short range velocity field that is due to the delta function type of forcing. In addition, as mentioned already, there is an exact analytical solution for this field (stokeslet) $[35] \mathbf{u}_{l}^{\mathcal{S}}(\mathbf{x})=\mathbf{H}_{l}\left(\mathbf{x}-\mathbf{r}^{k}\right) \cdot{ }^{d} \mathbf{F}^{k}$, hence it can readily be filtered $\overline{\mathbf{u}}_{l}^{\mathcal{S}}(\mathbf{x})=\int_{V} \mathbf{u}_{l}^{\mathcal{S}}\left(\mathbf{x}-\mathbf{x}^{\prime}\right) G_{\xi}\left(\mathbf{x}^{\prime}\right) \mathrm{d} \mathbf{x}^{\prime}$, obtain the residual velocity $\left(\mathbf{u}_{l}^{\mathcal{S}}\right)^{\prime}=\mathbf{u}_{l}^{\mathcal{S}}-\overline{\mathbf{u}}_{l}^{\mathcal{S}}$, and then filter the residual velocity tensor $\overline{\left(u_{l}^{\mathcal{S}}\right)_{i}^{\prime}\left(u_{l}^{\mathcal{S}}\right)_{j}^{\prime}}(\mathbf{x})=\int_{V}\left(u_{l}^{\mathcal{S}}\right)_{i}^{\prime}\left(\mathbf{x}-\mathbf{x}^{\prime}\right)\left(u_{l}^{\mathcal{S}}\right)_{j}^{\prime}\left(\mathbf{x}-\mathbf{x}^{\prime}\right) G_{\xi}\left(\mathbf{x}^{\prime}\right) \mathrm{d} \mathbf{x}^{\prime}$. Remarkably, since $\mathbf{u}_{l}^{\mathcal{S}}(\mathbf{x})$ decays exponentially on the length scale $\alpha^{-1}=\xi$, one can assume from the start that $\left(\mathbf{u}_{l}^{\mathcal{S}}\right)^{\prime} \approx \mathbf{u}_{l}^{\mathcal{S}}$ and avoid the intermediate step of filtering $\mathbf{u}_{l}^{\mathcal{S}}$. Finally, as 
discussed above, $\overline{u_{i}^{\prime} u_{j}^{\prime}}=\overline{\left(u_{l}^{\mathcal{S}}\right)_{i}^{\prime}\left(u_{l}^{\mathcal{S}}\right)_{j}^{\prime}}$, and the filtered momentum equation is therefore closed in an exact way. Notably, at first sight, it appears that the choice of $\mathbf{H}_{l}\left(\mathbf{x}-\mathbf{r}^{k}\right)$ introduces an ad-hoc element in the computation of $\overline{u_{i}^{\prime} u_{j}^{\prime}}$, hence, also, of the fluid-momentum. However, this is not the case, since altering $\mathbf{H}_{l}\left(\mathbf{x}-\mathbf{r}^{k}\right)$ affects (via hydrodynamic interactions) the polymer-fluid coupling force ${ }^{d} \mathbf{F}$ too, so that the total effect of these two terms on the dynamics of the fluid does not depend on the choice of the smoothing kernel. In this way, the algorithm for evolving the second key variable $\overline{\mathbf{u}}(\mathbf{x}, t)$ is also complete. This concludes the development of the method.

\section{Algorithmic information flow chart}

Based on the above, the information flow chart of the algorithm is as follows:

(1) Specify initial and boundary conditions for $\bar{u}_{i},\left(\mathbf{R}_{b}(t), \mathbf{R}_{e}(t)\right)$, and all other physical quantities.

(2) Increment time by the computational time step.

(3) Employing $\bar{u}_{i}$ and $\left(\mathbf{R}_{b}(t), \mathbf{R}_{e}(t)\right)$, solve the Stokes equations, and recover the subfilter, creeping flow field $u_{i}^{\mathcal{S}}$, that corresponds to the hydrodynamic interactions between chains.

(4) Employing the Stokes flow field $u_{i}^{\mathcal{S}}$, and the large scale flow field $\bar{u}_{i}$, update $\left(\mathbf{R}_{b}(t), \mathbf{R}_{e}(t)\right)$, and polymer related quantities.

(5) Employing the Stokes flow field $u_{i}^{\mathcal{S}}$, compute the residual stresses $\overline{u_{i}^{\prime} u_{j}^{\prime}}$ in the filtered Navier-Stokes equation.

(6) Employing the residual stresses $\overline{u_{i}^{\prime} u_{j}^{\prime}}$, update the filtered velocity field $\bar{u}_{i}$.

(7) Repeat step (2) until final time.

\section{EPILOGUE}

Turbulence and polymer physics are two of the most complex topics in statistical physics. Their combination in various applications (e.g., polymer drag reduction in boundary layers, grid turbulence, pipe or channel turbulent complex-fluid flows) requires powerful numerical and computational methods for technological development and design. The present paper 
provides an asymptotically exact algorithm for the calculation of such flows, that is physically complete, and can be employed to model flows of arbitrary polymer volume fractions and fluid inertia. Indeed, due to the accurate coupling of fluid and polymers/colloids, this method can be directly employed in the computation of laminar and unstable complex-fluid flows.

A key feature of the present approach is that the computation of the Stokesian flow field acts as a type of subfilter flow modeling that enables the (physically complete) evolution of both polymer chains and filtered velocity field. In this context, it is the large separation of time scales between diffusive creeping flow modes and inertial turbulence fluctuations that makes this subfilter flow modeling accurate. A quantitative measure of the validity of the scale-separation hypothesis is the ratio between the Reynolds numbers in the polymer $\left(R e_{p}\right)$ and Kolmogorov $\left(R e_{\eta}\right)$ range of scales, i.e. the smallest this number, the more justified the assumption of neglecting fluid inertia in the flow around the chains is, and the more accurate the calculations become. Employing the symbol $\eta$ for the Kolmogorov scale and $l_{p}$ for the typical polymer scale, and using as characteristic velocity the velocity fluctuation at the Kolmogorov scale, one obtains $R e_{p}=\left(l_{p} / \eta\right) R e_{\eta}$ (where $R e_{\eta} \approx 1$ ). In particular [24], for a turbulent flow in air with large-scale eddy velocity $u=2 \mathrm{~m} / \mathrm{s}$, and integral length scale $l=0.3 \mathrm{~m}$, it is $R e=u l / \nu \approx 0.5 \times 10^{5}$, and $\eta=0.1 \mathrm{~mm}$. On the other hand [21], for PEO polymer with molecular weight $M=1 \times 10^{6}$ Da, the smallest mesoscopic scale is Kuhn's (correlation) length $b_{K}=7.37 \times 10^{-7} \mathrm{~mm}$, and the largest is the (equilibrium) chain size $R_{c}=3 \times 10^{-4} \mathrm{~mm}$. Employing $l_{p}=b_{K}$ gives $R e_{p} / R e_{\eta}=7.37 \times 10^{-6}$, and $l_{p}=R_{c}$ gives $R e_{p} / R e_{\eta}=3 \times 10^{-3}$. Hence in both most favourable and adverse cases, $R e_{p} / R e_{\eta} \ll 1$, and the method has an asymptotic validity. It is important to note that, indeed, the equilibrium chain length $R_{c}$ is the largest polymer scale when it comes to Reynolds number considerations. This is not to ignore the fact that the flow can extend chains to much larger than $R_{c}$ lengths. However, the Reynolds number requires a length csale that characterizes the degree at which the chain appears like an obstacle in the flow, and, as Zimm theory of hydrodynamic interactions shows, the uniform (in the statistical sense) occupation of a certain fluid volume by the chain is required in order for the hydrodynamic interactions to couple the corresponding polymer parts together and make them appear like a solid object to the flow [1]. When a polymer extends, the chain is out of equilibrium and is not free to 
wander, hence the above effect is present only within Pincus tension blobs [1] of scale $l_{t}<R_{c}$ ( $l_{t}$ is the characteristic scale of the beads). Certainly, the above picture applies to dilute systems; as the system becomes dense, increasing polymer volume fraction is accompanied by decreasing hydrodynamic screening length, hence due to the fact that hydrodynamic interactions are screened over a larger range of scales, Zimm theory is replaced (at large scales) by Rouse theory, resulting in smaller $l_{p}$ values (that are more favourable in justifying the scale-separation hypothesis). Finally, it is important to note that the discussed scale-separation assumption is the only hypothesis of the formulation, and there are no other limitations regarding polymer volume fraction (since the algorithm models both single chains and polymer entanglements) or flow inertia (since the algorithm models both laminar and turbulent flows).

Certainly, computational complexity is of great concern with respect to the application of this method to realistic flows. Hence, the availability of parallel finite volume turbulent flow solvers, as well as the $O(N)$ character of the Stokesian dynamics method of [35] are important features. Additional algorithmic complexity improvements could be made in the future. Certainly, to a certain extent, computational modeling needs also to be applied. For example, one can start by having the polymers interact with an inertial range of limited extension in wavenumber space, or by reducing the number of beads per polymer chain. Indeed, the coarse-grained polymer physics model developed in [21] requires only material constants as input, and by automatically adjusting all its dynamical parameters (in a rational way) to the chosen degree of coarse-graining, it becomes very suitable for experiments with the degree of resolution of polymer dynamics or the specific polymer material employed. Definitely, complexity issues are intertwined with numerical stability issues and volume fraction levels. Regarding the former, and since the polymer time scales are much faster than the turbulent flow time scales, the time step is mostly restricted by polymer physics. For coarse-grained molecular dynamics, the latter dictate that the spring relaxation time must be resolved by the computation. For PEO polymers with molecular weight $M=1 \times 10^{6} \mathrm{Da}$ in a good solvent, the polymer model of [21] gives a spring relaxation time 100 times smaller that the diffusion time step of a finite volume solver that fully resolves velocity fluctuations in a turbulent flow with Taylor Reynolds number $R e_{\lambda}=70$. Phantom chain bead-spring computations done with the semi-implicit predictor corrector 
scheme of [41] are unconditionally stable. However, entangled chain dynamics algorithms implement the explicit midpoint method of Fixman [21], which needs a significantly smaller than the spring relaxation time step in order to avoid numerical instability due to polymer extension beyond the maximum chain length in flow areas of strong extensional stretching. Since turbulent flow solvers are highly optimized, it is not expected that the turbulent part of the computations is going to be a limiting factor in actual computations. On the other hand, the number of beads/particles (i.e. their volume fraction) required for the method to illustrate important physics will most certainly be. It is well known, that very small polymer concentrations of the order of a ten parts per million by weight can lead to polymer drag reduction phenomena. However, due to the computational complexity of polymer molecular dynamics, a direct numerical computation of actual experimental situations is not possible. Instead, significant experimentation with material properties of polymer chains and solvents, system size and flow field type would be necessary in order to delineate a threshold beyond which the polymers would start having a nontrivial effect in a particular flow. The latter would be a significantly easier task for the (computationally less complex) case of colloidal fluids, especially if the Brownian motion effects are switched off.

In another milieu, recently developed mesoscopic models of superfluid dynamics [42] have stressed the similarity of the latter with polymeric fluids. Because of this similarity, a variant of the present method could, in the future, be also applied to superfluid dynamics.

\section{ACKNOWLEDGEMENTS}

I am very grateful to Tony Leonard for valuable discussions, and Caltech overall for its generous hospitality during my visiting appointment.

[1] M. Rubinstein and R.H. Colby, Polymer Physics, Oxford University Press (2003).

[2] R.B. Bird, C.F. Curtiss, R.C. Armstrong, and O. Hassager, Dynamics of Polymeric Liquids, Volume 2: Kinetic Theory, Wiley-Interscience (1987).

[3] J. Mewis and N.J. Wagner, Colloidal Suspension Rheology, Cambridge University Press (2011). 
[4] W. Russel, Colloidal Dispersions, Cambridge University Press (1992).

[5] D.D. Joseph, Fluid Dynamics of Viscoelastic Liquids, Springer (2013).

[6] D. Joseph, T. Funada, and J. Wang, Potential Flows of Viscous and Viscoelastic Liquids, Cambridge University Press (2007).

[7] R.B. Bird, R.C. Armstrong, and O. Hassager, Dynamics of Polymeric Liquids, Volume 2: Fluid Mechanics, Wiley-Interscience (1987).

[8] C.D. Dimitropoulos, Y. Dubief E.S.G. Shaqfeh, and P. Moin, Direct numerical simulation of polymer-induced drag reduction in turbulent boundary layer flow of inhomogeneous polymer solutions, J. Fluid Mech. 566, 153 (2006).

[9] C.M. Casciola and E. De Angelis, Energy transfer in turbulent polymer solutions, J. Fluid Mech. 581, 419 (2007).

[10] S. Berti, A. Bistagnino, G. Boffetta, A. Celani, and S. Musacchio, Two-dimensional elastic turbulence, Phys. Rev E 77, 055306(R) (2008).

[11] R.I. Tanner, Engineering Rheology, Oxford University Press (2000).

[12] R.G. Larson, The Structure and Rheology of Complex Fluids, Oxford University Press (1999).

[13] L.G. Leal, Advanced Transport Phenomena: Fluid Mechanics and Convective Transport Processes, Cambridge University Press (2007).

[14] A. Tsinober, An Informal Conceptual Introduction to Turbulence, Springer (2009).

[15] U. Frisch, Turbulence: The Legacy of A. N. Kolmogorov, Cambridge University Press (1996).

[16] D. Kivotides and A. Leonard, Geometrical physics of the many vortex filament problem, EPL 66, 69 (2004).

[17] D. Kivotides, S.L. Wilkin, T.G. Theofanous, Stretching of polymer chains by fluctuating flow fields, Phys. Lett. A 375, 48 (2010).

[18] S. Jin, L.R. Collins, Dynamics of dissolved polymer chains in isotropic turbulence, New J. Phys. 9 (2007) 360.

[19] P. Oswald, Rheophysics: The Deformation and Flow of Matter, Cambridge University Press (2009).

[20] D. Kivotides, S.L. Wilkin, T.G. Theofanous, Entangled chain dynamics of polymer knots in extensional flow, Phys. Rev. E 80, 041808 (2009).

[21] D. Kivotides, S.L. Wilkin, T.G. Theofanous, Stochastic entangled chain dynamics of dense polymer solutions, J. Chem. Phys. 133, 144903 (2010). 
[22] R. Vonlanthen, P. A. Monkewitz, Grid turbulence in dilute polymer solutions: PEO in water, J. Fluid Mech. 730, 76 (2013).

[23] M.D. Graham, Drag reduction in turbulent flow of polymer solutions, Rheology Reviews 2004, pp 143-170.

[24] P.A. Davidson, turbulence, OUP (2004).

[25] S.K. Friendlander, Smoke, Dust, and Haze: Fundamentals of Aerosol Dynamics, Oxford University Press (2000).

[26] B.K. Brunk, D.L. Koch and L.W. Lion, Turbulent coagulation of colloidal particles, J. Fluid Mech. 364, 81 (1998).

[27] J. Chun, D. Koch, S. Rani, A. Ahluwalia and L. Collins, Clustering of aerosol particles in isotropic turbulence, J. Fluid Mech. 536, 219 (2005).

[28] P. Saffman, and J. Turner, On the collision of drops in turbulent clouds, J. Fluid Mech. 1, 16 (1956).

[29] G. Falkovich, A. Fouxon, and M.G. Stepanov, Acceleration of rain initiation by cloud turbulence, Nature 419, 151 (2002).

[30] P.K. Wang, Physics and Dynamics of Clouds and Precipitation, Cambridge University Press (2013).

[31] A. Prosperetti and G. Tryggvason (Eds), Computational Methods for Multiphase Flow, Cambridge University Press (2007).

[32] T. Padding and W. J. Briels, Uncrossability constraints in mesoscopic polymer melt simulations: Non-Rouse behavior of C120H242, J. Chem. Phys. 115, 2846 (2001).

[33] E. G. Sinaiski and L.I. Zaichik, Statistical Microhydrodynamics, Wiley-VCH (2008).

[34] A.J. Banchio and J.F. Brady, Accelerated Stokesian Dynamics: Brownian Motion, J. Chem. Phys. 118, 10323 (2003).

[35] J.P. Hernandez-Ortiz, J.J. de Pablo and M. D. Graham, Fast Computation of Many-Particle Hydrodynamic and Electrostatic Interactions in a Confined Geometry, Phys. Rev. Lett. 98, 140602 (2007).

[36] P. Orlandi, Fluid Flow Phenomena: A Numerical Toolkit, Springer (2006).

[37] D. Kivotides, Relaxation of superfluid vortex bundles via energy transfer to the normal fluid, Phys. Rev. B 76, 054503 (2007). 
[38] Y. Zhang, J.J. de Pablo and M.D. Graham, An immersed boundary method for Brownian dynamics simulation of polymers in complex geometries: Application to DNA flowing through a nanoslit with embedded nanopits, J. Chem. Phys. 136, 014901 (2012).

[39] M. Fixman, Simulation of polymer dynamics. I. General theory, J. Chem. Phys. 69, 1527 (1978).

[40] P. Grassia, E. Hinch and L. Nitsche, Computer simulations of Brownian motion of complex systems, J. Fluid Mech. 282, 373 (1995).

[41] M. Somasi, B. Khomami, N.J. Woo, J.S. Hur, E.S.G. Shaqfeh, Brownian dynamics simulations of bead-rod and bead-spring chains: numerical algorithms and coarse-graining issues, J. NonNewtonian Fluid Mech. 108, 227 (2002).

[42] D. Kivotides, Energy spectra of finite temperature superfluid helium-4 turbulence, Phys. Fluids 26, 105105 (2014). 